\title{
FINE STRUCTURES INSIDE THE PRELIE OPERAD
}

\author{
FRÉDÉRIC CHAPOTON
}

(Communicated by Jim Haglund)

\begin{abstract}
This article aims at a detailed analysis of the PreLie operad. We obtain a concrete description (as a morphism) of the relationship between the anticyclic structure of PreLie and the generators of PreLie as a Lie-module, which was previously known only at the level of characters. Building on this, we obtain a surprising inclusion of the cyclic Lie module in the PreLie operad. We conjecture that the image of this inclusion generates an interesting free sub-operad.
\end{abstract}

\section{INTRODUCTION}

The aim of this article is to make a step forward in the study of a specific operad, called the PreLie operad, and to unravel some unexpected relations with the Lie operad. The PreLie operad describes pre-Lie algebras, which have been useful in various contexts and were introduced independently in the works of Gerstenhaber [10] in deformation theory and in the works of Vinberg [18] on convex homogeneous cones. The PreLie operad itself has been described in [6] in terms of combinatorial objects called rooted trees. This description is strongly connected to the use of rooted trees in numerical analysis [3], because the space of vector fields on the affine space is naturally a pre-Lie algebra.

Many results are already known on the PreLie operad. It was proved in [6] that it is a Koszul operad, and this result has been re-proved since then by other methods [17, 12. Another important property is the fact, first proved in [9], that free pre-Lie algebras are free Lie algebras, where the Lie bracket comes from antisymmetrising the pre-Lie product. Another proof has been obtained in [5] in the setting of operads rather than algebras. This property translates into the fact that the PreLie operad is a free left Lie-module, so that there exists a factorisation

$$
\text { PreLie }=\text { Lie } \circ X
$$

in the category of $\mathfrak{S}$-modules, where $\circ$ is the composition of $\mathfrak{S}$-modules. Our principal aim is to better understand the $\mathfrak{S}$-module $X$.

Surprisingly enough, the $\mathfrak{S}$-module $X$ is related to another structure on the PreLie operad. It was shown in [4 that PreLie is an anticyclic operad. This implies in particular that the $\mathfrak{S}$-module PreLie admits a primitive CycPreLie: this means that the $\mathfrak{S}_{n+1}$-module $\operatorname{CycPreLie}(n+1)$ gives, by restriction of the action, a module over the symmetric group $\mathfrak{S}_{n}$ which is isomorphic to $\operatorname{PreLie}(n)$. It has

Received by the editors December 12, 2011 and, in revised form, January 17, 2012. 2010 Mathematics Subject Classification. Primary 05C05, 18D50; Secondary 17A30. 
been proved in [5, Th. 5.3] that for $n \geq 2$ there is an isomorphism of modules over the symmetric group $\mathfrak{S}_{n}$,

$$
X(n) \simeq \operatorname{Reflex}(n) \otimes \operatorname{CycPreLie}(n),
$$

where $\operatorname{Reflex}(n)=\mathbb{Q}^{n} / \mathbb{Q}$ is the quotient of the natural action of $\mathfrak{S}_{n}$ on $\mathbb{Q}^{n}$ by the diagonal subspace. This was proved using explicit formulas for the characters of these modules. The first main result of the present article is to better explain this relation by the definition of an explicit isomorphism. This will be achieved in section 7 .

The other main result of the article is the following. It is well known that the Lie operad is a cyclic operad [13, so that in particular there exists a primitive CycLie to the $\mathfrak{S}$-module Lie. We obtain, using the previous parts of the article, an injective morphism of $\mathfrak{S}$-modules from CycLie to PreLie. This is a surprising result, which relates classical operads in a rather strange way.

We expect that this inclusion of CycLie into PreLie should moreover have very nice properties. We conjecture that it generates a free sub-operad of PreLie. A recent result by N. Bergeron and Loday [2] would be a direct corollary of this more general conjecture. Moreover, we conjecture that the sub-operad of PreLie generated by the image of CycLie should be isomorphic to $X$ as a $\mathfrak{S}$-module and should give a distinguished space of generators of PreLie as a Lie-module.

\section{General Setting And notation}

We have chosen for concreteness to work over the ground field $\mathbb{Q}$, but all statements and their proofs hold true over any field of zero characteristic.

We will use the description of operads using $\mathfrak{S}$-modules over $\mathbb{Q}$. The reader may want to consult [15] for further details on the theory of operads.

Recall that the category of $\mathfrak{S}$-modules over $\mathbb{Q}$ is the category of functors from the groupoid of finite sets to the category of $\mathbb{Q}$-vector spaces. By choosing an equivalent model for the groupoid of finite sets, $\mathfrak{S}$-modules can also be described as collections of vector spaces $P(n)$ with an action of the symmetric group $\mathfrak{S}_{n}$ on $P(n)$ for $n \geq 0$. We will freely use both descriptions. The index $n$ will be called the arity.

We will denote by $S_{1}$ the $\mathfrak{S}$-module corresponding to the trivial representation of $\mathfrak{S}_{1}$.

The derivative $P^{\prime}$ of an $\mathfrak{S}$-module $P$ is defined as follows. For every $n$, the space $P^{\prime}(n-1)$ is $P(n)$, with the action of the symmetric group $\mathfrak{S}_{n-1}$ given by restriction of the action of the symmetric group $\mathfrak{S}_{n}$ on $P(n)$. A primitive of a $\mathfrak{S}$-module $P$ is a S-module $Q$ such that $Q^{\prime}=P$.

The category of $\mathfrak{S}$-modules is endowed with a nonsymmetric tensor product $\circ$ which is called the composition. An operad is a monoid for this tensor product. We will also consider right and left modules over operads for the same monoidal structure.

We will also need the symmetric tensor structure $\otimes$ on the category of $\mathfrak{S}$-modules defined by

$$
(P \otimes Q)(I)=\bigoplus_{I=J \sqcup K} P(J) \otimes Q(K) .
$$

Using this tensor product, one can also define exterior and symmetric powers of S-modules. 
There is a third tensor product on the category of $\mathfrak{S}$-modules, sometimes called the Hadamard product, defined by

$$
(P \odot Q)(I)=P(I) \otimes Q(I) .
$$

There is an equivalent way to describe an operad $\mathcal{P}$, using partial compositions instead of the global composition map $\mathcal{P} \circ \mathcal{P} \rightarrow \mathcal{P}$. We will denote by $\circ_{*}$ the partial composition at position $*$ in an operad. Symbols like $*$, \# will sometimes appear as placeholders for positions of compositions and will always be assumed to be distinct from any other index.

For the definition and basic statements about cyclic operads, the reader can for example look at the foundational article [1] or at [7, 14].

\section{THE Lie OPERAD}

Recall that a Lie algebra is a vector space $V$ endowed with an antisymmetric bracket $(x, y) \mapsto[x, y]$ such that

$$
[x,[y, z]]+[x,[y, z]]+[x,[y, z]]=0 .
$$

This relation is called the Jacobi identity.

Let Lie be the operad describing Lie algebras.

The operad Lie admits a presentation by generators and relations, which amounts to the axiomatic description of Lie algebras given above. There is an antisymmetric generator in two variables, and there is a relation between compositions of two generators, given by (1). if

Recall now that a symmetric bilinear form ( , ) on a Lie algebra is called invariant

$$
([x, y], z)=(x,[y, z]) .
$$

This invariance condition is classical and comes from the natural invariance condition for bilinear forms under group actions.

This notion of an invariant bilinear form leads to a structure of cyclic operad on Lie, first introduced by Kontsevich [13. The S-module CycLie (cyclic Lie module) is the right Lie-module defined by the exact sequence of right Lie-modules,

$$
K_{L} \rightarrow S^{2} \text { Lie } \stackrel{(,)}{\longrightarrow} \text { CycLie } \rightarrow 0,
$$

where $K_{L}$ is the sub-right Lie-module of $S^{2}$ Lie generated by the relation (2).

As an S-module, CycLie is a primitive of Lie.

The following statement is a general property of cyclic operads.

Proposition 2.1. Let I be a finite set. For every element $\ell$ in $\mathrm{CycLie}(I)$ and every element $i \in I$, there is a unique element $\ell_{i}$ in $\operatorname{Lie}(I \backslash\{i\})$ such that

$$
\ell=\left(i, \ell_{i}\right) \text {. }
$$

For example, if $\ell=([x, y], z)$ and $i=x$, then $\ell_{i}=[y, z]$ by (2).

\section{The PreLie OPERAD}

Recall that a pre-Lie algebra is a vector space $V$ endowed with a bilinear map $(x, y) \mapsto x \triangleleft y$ such that

$$
(x \triangleleft y) \triangleleft z-x \triangleleft(y \triangleleft z)=(x \triangleleft z) \triangleleft y-x \triangleleft(z \triangleleft y) .
$$

Let PreLie be the operad describing pre-Lie algebras. 
The operad PreLie admits a combinatorial description using rooted trees [6]. Let us recall this briefly here.

For a finite set $I$, a rooted tree on $I$ is a connected and simply connected graph with vertex set $I$, together with a distinguished vertex called the root. One can then orient every edge towards the root. Then PreLie $(I)$ is the vector space spanned by rooted trees on $I$. The composition of two rooted trees $S \circ_{i} T$ is the sum of all rooted trees obtained from the disjoint union of $S \backslash\{i\}$ and $T$ by adding one edge for every edge that was incident to $i$ in $S$, as follows. Edges that were incoming at $i$ must keep the same start and end at some vertex of $T$. The edge that was outgoing at $i$ (if it exists) must keep the same end and start at the root of $T$. The root of every tree in the sum is the unique vertex with no outgoing edge.

The operad PreLie admits a presentation by generators and relations, which amounts to the axiomatic description of pre-Lie algebras given above. There is a generator in two variables, and there is a relation between compositions of two generators, given by (5).

From the description by rooted trees of the operad PreLie given above, one can get the following rule for the product $S \triangleleft T$ of two rooted trees $S$ and $T$ : this is the sum of all rooted trees obtained from the disjoint union of $S$ and $T$ by adding one edge from the root of $T$ to a vertex of $S$. The root of each tree in this sum is the root of $S$.

Recall now [4, §5.3] that an antisymmetric bilinear form $\langle$,$\rangle on a pre-Lie algebra$ is called invariant if

$$
\langle x, y \triangleleft z\rangle=-\langle z, y \triangleleft x\rangle
$$

and

$$
\langle x, y \triangleleft z\rangle=\langle y, x \triangleleft z-z \triangleleft x\rangle .
$$

This invariance condition is less classical than its Lie analogue, but has been used in the study of left-invariant affine and symplectic structures on Lie groups. It is also related to the notion of a quasi-Frobenius Lie algebra.

Note that (6) is a consequence of (7) and that these relations also imply the following relation:

$$
\langle x, y \triangleleft z\rangle+\langle y, z \triangleleft x\rangle+\langle z, x \triangleleft y\rangle=0 .
$$

This notion of an invariant bilinear form leads to a structure of anticyclic operad on PreLie, introduced in 4 . The anticyclic $\mathfrak{S}$-module CycPreLie is the right PreLiemodule defined by the exact sequence of right PreLie-modules

$$
K_{P} \rightarrow \Lambda^{2} \text { PreLie } \stackrel{\langle,\rangle}{\longrightarrow} \text { CycPreLie } \rightarrow 0,
$$

where $K_{P}$ is the sub-right PreLie-module of $\Lambda^{2}$ PreLie generated by the relations (6) and (7).

As an S-module, CycPreLie is a primitive of PreLie.

The following statement is a general property of anticyclic operads.

Proposition 3.1. Let $I$ be a finite set. For every $t \in \operatorname{CycPreLie}(I)$ and every $i \in I$, there is a unique $\Gamma_{i}(t) \in \operatorname{PreLie}(I \backslash\{i\})$ such that

$$
t=\left\langle i, \Gamma_{i}(t)\right\rangle \text {. }
$$


For example, if $t=\langle x, y \triangleleft z\rangle$, then $\Gamma_{z}(t)=-y \triangleleft x$ by (6).

Here is a more complicated example. If $t=\langle w, x \triangleleft(y \triangleleft z)\rangle$, then one can show that $\Gamma_{z}(t)=-y \triangleleft(x \triangleleft w)$.

Let us now state some lemmas for later use. For short, we will write $a \in s$ as an abbreviation for the sentence $s \in \operatorname{PreLie}(I)$ and $a \in I$ for some finite set $I$. We will also say that $r$ and $s$ have disjoint indices if $r \in \operatorname{PreLie}(I)$ and $s \in \operatorname{PreLie}(J)$ for some disjoint finite sets $I$ and $J$.

Lemma 3.2. Let $r, s, t$ be in PreLie with disjoint indices. Let $a \in s$ and $b \in r \sqcup s$ be distinct from $a$. Then

$$
\Gamma_{b}\left(\left\langle r, s \circ_{a} t\right\rangle\right)=\Gamma_{b}(\langle r, s\rangle) \circ_{a} t .
$$

Proof. Indeed, one has

$$
\begin{aligned}
\left\langle b, \Gamma_{b}\left(\left\langle r, s \circ_{a} t\right\rangle\right)\right\rangle=\left\langle r, s \circ_{a} t\right\rangle & =\langle r, s\rangle \circ_{a} t \\
& =\left\langle b, \Gamma_{b}(\langle r, s\rangle)\right\rangle \circ_{a} t=\left\langle b, \Gamma_{b}(\langle r, s\rangle) \circ_{a} t\right\rangle .
\end{aligned}
$$

Here one uses the definition of $\Gamma$ and the fact that CycPreLie is a right PreLiemodule.

Lemma 3.3. Let $r, s, t$ be in PreLie with disjoint indices. Let $a \in s$ and $b \in t$. Then

$$
\Gamma_{b}\left(\left\langle r, s \circ_{a} t\right\rangle\right)=-\Gamma_{b}(\langle \#, t\rangle) \circ_{\#} \Gamma_{a}(\langle r, s\rangle),
$$

where \# is a placeholder symbol, distinct from indices of $r, s, t$.

Proof. Let us compute $\langle \#, t\rangle \circ_{\#} \Gamma_{a}(\langle r, s\rangle)$ in two ways. On the one hand, this is equal by definition of $\Gamma$ to

$$
\left\langle b, \Gamma_{b}(\langle \#, t\rangle)\right\rangle \circ_{\#} \Gamma_{a}(\langle r, s\rangle),
$$

which can be rewritten, by the right module property, as

$$
\left\langle b, \Gamma_{b}(\langle \#, t\rangle) \circ_{\#} \Gamma_{a}(\langle r, s\rangle)\right\rangle .
$$

On the other hand, this is equal by properties of $\Gamma$ to

$$
\left\langle\Gamma_{a}(\langle r, s\rangle), t\right\rangle=\left\langle\Gamma_{a}(\langle r, s\rangle), a\right\rangle \circ_{a} t,
$$

which by definition of $\Gamma$ is

$$
-\langle r, s\rangle \circ_{a} t=-\left\langle r, s \circ_{a} t\right\rangle=-\left\langle b, \Gamma_{b}\left(\left\langle r,\left(s \circ_{a} t\right)\right\rangle\right)\right\rangle .
$$

This proves the expected equality.

\section{PreLie IS FREe AS A Lie-MOdule}

Recall that every pre-Lie algebra is also a Lie algebra for the bracket defined by

$$
[x, y]=x \triangleleft y-y \triangleleft x .
$$

This defines a morphism $\varphi$ of operads from Lie to PreLie. The composition with the projection from PreLie to the associative operad Assoc is the usual inclusion of Lie in Assoc; hence $\varphi$ is also injective.

From this morphism, one can deduce by restriction of composition in PreLie a structure of left Lie-module on PreLie:

$$
\text { Lie } \circ \text { PreLie } \stackrel{\gamma}{\longrightarrow} \text { PreLie . }
$$

It is clear from this definition that $\gamma$ is a morphism of right PreLie-modules. 
Let $\mathrm{Lie}_{\geq 2}$ be the restriction of Lie to arities at least 2. One can restrict $\gamma$ to Lie $_{\geq 2} \circ$ PreLie and define a $\mathfrak{S}$-module Indec by the exact sequence of right PreLiemodules

$$
\operatorname{Lie}_{\geq 2} \circ \text { PreLie } \stackrel{\gamma}{\longrightarrow} \text { PreLie } \stackrel{\pi}{\longrightarrow} \text { Indec } \rightarrow 0 .
$$

The $\mathfrak{S}$-module Indec was denoted by $X$ in the introduction.

It has been shown in [5] (see also [9, 1]) that PreLie is a free left Lie-module: there exists an isomorphism of $\mathfrak{S}$-modules

$$
\text { PreLie } \simeq \text { Lie } \circ \text { Indec } .
$$

This isomorphism is not canonical, but depends on the choice of a section of the projection $\pi$.

This statement of freeness can be reformulated as follows. The left Lie-module structure of PreLie can be considered as a structure of Lie-algebra in the category of $\mathfrak{S}$-modules (with respect to the tensor product $\otimes$ ). The usual theory of Lie algebras over a field has a natural extension to this setting, including universal enveloping algebras and the Chevalley-Eilenberg complex (see for instance [16]). Freeness as a left Lie-module then translates into freeness as a Lie algebra, which implies that the Chevalley-Eilenberg complex has homology only in degree 0 .

As the image of the leftmost arrow $\gamma$ of (16) is spanned by the linear combinations of brackets of rooted trees, there is a long exact sequence of right PreLie-modules

$$
\cdots \rightarrow \Lambda^{3} \text { PreLie } \stackrel{\delta}{\longrightarrow} \Lambda^{2} \text { PreLie } \stackrel{\delta}{\longrightarrow} \text { PreLie } \stackrel{\pi}{\longrightarrow} \text { Indec } \rightarrow 0,
$$

where $\delta$ are the Chevalley-Eilenberg differentials. The rightmost $\delta$ sends $s \wedge t$ to $s \triangleleft t-t \triangleleft s$.

This gives a short exact sequence of right PreLie-modules

$$
0 \rightarrow \text { Rela } \stackrel{\delta}{\longrightarrow} \text { PreLie } \stackrel{\pi}{\longrightarrow} \text { Indec } \rightarrow 0,
$$

where Rela is the quotient of $\Lambda^{2}$ PreLie by the image of $\delta$.

Proposition 4.1. The right PreLie-module Rela is the quotient of $\Lambda^{2}$ PreLie by the sub-right PreLie-module generated by the following 6-term relations:

$(20) r \wedge(s \triangleleft t)+s \wedge(t \triangleleft r)+t \wedge(r \triangleleft s)-s \wedge(r \triangleleft t)-t \wedge(s \triangleleft r)-r \wedge(t \triangleleft s)=0$.

Proof. We are dealing here with the first few terms of the Chevalley-Eilenberg complex for the Lie algebra PreLie. As we know that this is a free Lie algebra, there is no homology but in degree 0 . This implies that the kernel is spanned by the image of $r \wedge s \wedge t$ by $\delta$ :

$$
[r, s] \wedge t+[s, t] \wedge r-[r, t] \wedge s .
$$

Using the link between the bracket and $\triangleleft$, this gives the expected relations.

\section{Reduction to Root-VAlence 1}

Let us define the root-valence of a rooted tree $T$ to be the valence of the root of $T$, i.e. the number of edges adjacent to the root.

Recall the following standard notation: for rooted trees $T_{1}, \ldots, T_{k}$, let

$$
B_{a}^{+}\left(T_{1}, T_{2}, \ldots, T_{k}\right)
$$

be the rooted tree obtained by grafting $T_{1}, \ldots, T_{k}$ on a new root with index $a$. 
If $S$ is a rooted tree in PreLie, we will denote by $[S]$ the class of $S$ modulo the image of $\delta$, i.e. modulo Lie brackets of rooted trees.

Proposition 5.1. For all rooted trees $S$ and $T$ and $* \in S$, one has

$$
\left[S \circ_{*} T\right]=[S] \circ_{*} T .
$$

Proof. This is because (19) is an exact sequence of right PreLie-modules.

Theorem 5.2. Every rooted tree $T$ in PreLie with at least two vertices is equivalent modulo $\delta\left(\Lambda^{2}\right.$ PreLie) to a linear combination of rooted trees of root-valence 1.

The proof is by induction on the size of rooted trees and uses several lemmas of increasing generality.

Lemma 5.3. The statement is true if $T$ has root-valence 2 and at least one of the two subtrees of the root is a singleton a.

Proof. Let $T^{\prime}$ be the tree $T$ with vertex $a$ removed. Then $T^{\prime}$ has root-valence 1 . Then one has $\left[T^{\prime}, a\right]=T^{\prime} \triangleleft a-a \triangleleft T^{\prime}$. The tree $a \triangleleft T^{\prime}$ has root-valence 1 . One has $T^{\prime} \triangleleft a=T+r$, where $r$ is a sum of trees of root-valence 1 .

Lemma 5.4. The statement is true if $T$ has a leaf a such that the vertex $b$ under a has valence 1 .

Proof. If $T$ is the rooted tree $b \triangleleft a$, the statement is true. Otherwise, one can write $T=T^{\prime} \circ_{*}(b a)$, where $T^{\prime}$ is a smaller tree (with at least two vertices) with a leaf $*$ and where $(b a)$ is the rooted tree $b \triangleleft a$. By induction on the size, the tree $T^{\prime}$ is equivalent to a linear combination $\sum_{\alpha} \mathbf{c}_{\alpha} T_{\alpha}$ of trees of root-valence 1. By Proposition 5.1, the tree $T$ is equivalent to the linear combination $\sum_{\alpha} \mathbf{c}_{\alpha} T_{\alpha} \circ_{*}(b a)$.

If $*$ is not the root of $T_{\alpha}$, then $T_{\alpha} \circ_{*}(b a)$ is a sum of trees of root-valence 1 . If $*$ is the root of $T_{\alpha}$, then $T_{\alpha} \circ_{*}(b a)$ is the sum of a rooted tree of root-valence 1 plus a rooted tree of root-valence 2 , which satisfies the hypothesis of Lemma 5.3

Lemma 5.5. The statement is true if at most one of the subtrees of the root of $T$ is not a leaf.

Proof. By induction on the root-valence $k$. If $k=1$, there is nothing to prove. If $k=2$, we can use Lemma 5.3 above.

Assume now that $k$ is at least 2 and write $T=B_{a}^{+}\left(T_{1}, a_{2}, \ldots, a_{k}\right)$.

Let $T^{\prime}=B_{a}^{+}\left(T_{1}, a_{2}, \ldots, a_{k-1}\right)$ be the tree obtained from $T$ by removing the leaf $a_{k}$. Then $\left[T^{\prime}, a_{k}\right]=T^{\prime} \triangleleft a_{k}-a_{k} \triangleleft T^{\prime}$. The tree $a_{k} \triangleleft T^{\prime}$ has root-valence 1 . One has $T^{\prime} \triangleleft a_{k}=T+r$, where $r$ is a sum of two kinds of trees: either $a_{k}$ is grafted on one of the leaves $a_{i}$, in which case one can apply Lemma [5.4, or $a_{k}$ is grafted on $T_{1}$, in which case one can use the induction on $k$.

Lemma 5.6. The statement is true for any rooted tree $T$ with at least 2 vertices.

Proof. Pick in $T$ a vertex $a$ of maximal height, where the height of a vertex is the number of edges in the unique path to the root. Let $b$ be the vertex under $a$. The set of all vertices over $b$ is then a corolla $C_{b}$.

If $T$ is just the corolla $C_{b}$, the statement is true by Lemma 5.5. Let us therefore assume that this is not the case.

In this case, $T$ can be written $T^{\prime} \circ_{*} C_{b}$, where $T^{\prime}$ has at least 2 vertices. By induction on the size, $T^{\prime}$ is equivalent to a linear combination $\sum_{\alpha} \mathbf{c}_{\alpha} T_{\alpha}$ of trees of root-valence 1 . 
By Proposition 5.1, $T$ is equivalent to the linear combination $\sum_{\alpha} \mathbf{c}_{\alpha} T_{\alpha} \circ_{*} C_{b}$.

If $*$ is not the root of $T_{\alpha}$, then $T_{\alpha} \circ_{*} C_{b}$ is a sum of trees of root-valence 1 . If $*$ is the root of $T_{\alpha}$, then $T_{\alpha} \circ_{*} C_{b}$ is a sum of rooted trees, all satisfying the hypothesis of Lemma 5.5 .

This concludes the proof of Proposition 5.1.

Remark 5.7. What we used in the proof of Lemma 5.6 is a top corolla, i.e. a vertex $b$ with only leaves above it. Instead of choosing one such vertex of maximal height, it may be more convenient for purposes of practical computation of $\rho$ to choose one with the smallest number of attached leaves.

Let $\operatorname{PreLie}_{v=1}$ be the sub-S-module of PreLie spanned by rooted trees with root-valence 1 .

Remark 5.8. The S-module PreLie ${ }_{v=1}$ is not a right PreLie-module.

Let Rela ${ }_{v=1}$ be the subspace of Rela that is mapped by $\delta$ to $\operatorname{PreLie}_{v=1}$.

Let Indec $\geq 2$ be the sub-S-module of Indec obtained by removing the arity 1 component of Indec.

From Theorem 5.2 and the short exact sequence (19), one obtains a short exact sequence of $\mathfrak{S}$-modules

$$
0 \rightarrow \operatorname{Rela}_{v=1} \stackrel{\delta}{\longrightarrow} \operatorname{PreLie}_{v=1} \stackrel{\pi}{\longrightarrow} \text { Indec }_{\geq 2} \rightarrow 0 .
$$

Let us now describe another short exact sequence and then compare them.

\section{A simple Short EXACT SEQUenCe}

Let Comm be the underlying $\mathfrak{S}$-module of the commutative operad. For every finite set $I, \operatorname{Comm}(I)=\mathbb{Q}$.

Let Perm be the underlying $\mathfrak{S}$-module of the permutative operad. For every finite set $I, \operatorname{Perm}(I)=\mathbb{Q} I$.

There is an inclusion $\iota$ from Comm to Perm that sends $1 \in \operatorname{Comm}(I)$ to $\sum_{i \in I} i \in$ $\operatorname{Perm}(I)$.

Let Reflex be the quotient $\mathfrak{S}$-module, so that there is a short exact sequence

$$
0 \rightarrow \text { Comm } \stackrel{\iota}{\longrightarrow} \text { Perm } \stackrel{p}{\longrightarrow} \text { Reflex } \rightarrow 0 .
$$

By the (Hadamard) tensor product with CycPreLie, one gets a short exact sequence

$$
0 \rightarrow \text { CycPreLie } \stackrel{\iota}{\longrightarrow} \text { Perm } \odot \text { CycPreLie } \stackrel{p}{\longrightarrow} \text { Reflex } \odot \text { CycPreLie } \rightarrow 0 .
$$

There exists a section of the projection map $p$ in the short exact sequence (24) that maps the class of $i-j$ to $i-j$. This gives a similar section of the projection $p$ in the short exact sequence (25).

\section{ISOMORPHISM OF EXACT SEQUENCES}

In this section, we will obtain the following isomorphism of short exact sequences:

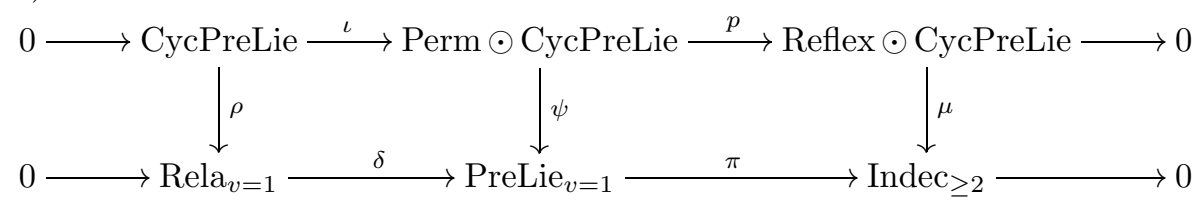


7.1. Middle column. Let us start with the isomorphism between middle terms. There is a simple isomorphism $\psi$ from Perm $\odot$ CycPreLie to PreLie ${ }_{v=1}$ defined for $t$ in CycPreLie and $a \in t$ by

$$
\psi(a \otimes t)=a \triangleleft \Gamma_{a}(t),
$$

where $\Gamma_{a}(t)$ is the function introduced in Proposition 3.1 .

The inverse morphism maps a rooted tree of root-valence 1, written as $a \triangleleft T$, to the expression $a \otimes\langle a, T\rangle$.

Note that the composite morphism $\psi \iota$ therefore has the following description:

$$
\psi(\iota(t))=\sum_{j} j \triangleleft \Gamma_{j}(t) .
$$

\subsection{Left column (up).}

Proposition 7.1. There exists a morphism $\langle$,$\rangle from Rela to CycPreLie.$

Proof. Let us start with the morphism $\langle$,$\rangle from \Lambda^{2}$ PreLie to CycPreLie that defines the anticyclic structure. By Proposition 4.1, it is enough to check that the 6-term relations (20) are mapped to 0. These relations are mapped in CycPreLie to

$$
\langle r, s \triangleleft t\rangle+\langle s, t \triangleleft r\rangle+\langle t, r \triangleleft s\rangle-\langle s, r \triangleleft t\rangle-\langle t, s \triangleleft r\rangle-\langle r, t \triangleleft s\rangle .
$$

Using (8) twice, one obtains that this vanishes in CycPreLie.

By restriction of the morphism $\langle$,$\rangle from Rela to CycPreLie, one has a morphism$ from Rela ${ }_{v=1}$ to CycPreLie, still denoted $\langle$,$\rangle .$

\subsection{Left column (down).}

Theorem 7.2. There exists a unique morphism $\rho$ from CycPreLie to Rela ${ }_{v=1}$ such that $\delta \rho$ equals $\psi \iota$, i.e.

$$
\delta(\rho(x))=\sum_{i} i \triangleleft \Gamma_{i}(x) .
$$

The morphism $\rho$ has the following property:

$$
\rho\left(\langle r, s\rangle \circ_{i} t\right)=\rho(\langle r, s\rangle) \circ_{i} t-\rho(\langle \#, t\rangle) \circ_{\#} \Gamma_{i}(\langle r, s\rangle)+\Gamma_{i}(\langle r, s\rangle) \wedge t,
$$

for $r, s, t$ in PreLie with disjoint indices and $i \in r \sqcup s$.

Proof. Let us start by remarking that the uniqueness of $\rho$ is clear because $\delta$ is an injection.

Assuming now for a moment that $\rho$ has been defined, let us prove the last statement, using uniqueness. Let us apply $\delta$ to the right side of (30):

$$
\sum_{j \in r, s}\left(j \triangleleft \Gamma_{j}(\langle r, s\rangle)\right) \circ_{i} t-\sum_{j \in \# \sqcup t}\left(j \triangleleft \Gamma_{j}(\langle \#, t\rangle)\right) \circ_{\#} \Gamma_{i}(\langle r, s\rangle)+\left[\Gamma_{i}(\langle r, s\rangle), t\right] .
$$

The term with $j=i$ in the first sum and the term with $j=\#$ in the second sum annihilates with the bracket term. One can then use Lemma 3.3 to rewrite the second sum and obtain

$$
\sum_{\substack{j \in r, s \\ j \neq i}} j \triangleleft \Gamma_{j}\left(\langle r, s\rangle \circ_{i} t\right)+\sum_{j \in t} j \triangleleft \Gamma_{j}\left(\langle r, s\rangle \circ_{i} t\right),
$$

which is exactly $\psi\left(\iota\left(\langle r, s\rangle \circ_{i} t\right)\right)$. 
Remark 7.3. One can also interpret this argument as follows: if equation (29) is satisfied by the terms entering the right-hand side of (30), then it is also satisfied by the left-hand side.

Let us now enter the existence proof of $\rho$.

It is enough to define a morphism from $\Lambda^{2}$ PreLie to Rela $v_{v=1}$ satisfying (29), as it will then automatically pass to the quotient CycPreLie.

As the set of elements $T \wedge T^{\prime}$ (for rooted trees $T$ and $T^{\prime}$ with disjoint indices) spans $\Lambda^{2}$ PreLie, it is sufficient to define $\rho\left(T \wedge T^{\prime}\right)$.

The definition is by induction on the cardinality of the finite set $I$ and, at fixed cardinality, by a four-step process for pairs of trees of increasing generality.

First step. Let us define $\rho$ when $I$ has cardinality 2 or 3 as

$$
\rho(a \wedge b)=a \wedge b
$$

and

$$
\rho(a \wedge(b \triangleleft c))=a \wedge(b \triangleleft c)-c \wedge(b \triangleleft a) .
$$

One can easily check that indeed $\delta \rho=\psi \iota$ in these cases.

Second step. Assume now that $T=a$ and that $T^{\prime}=b \triangleleft T^{\prime \prime}$ where $T^{\prime \prime}$ has at least two vertices.

One then defines $\rho\left(T \wedge T^{\prime}\right)$ using induction on the number of vertices by the following formula:

$\rho\left(a \wedge\left(b \triangleleft T^{\prime \prime}\right)\right)=\rho(a \wedge(b \triangleleft *)) \circ_{*} T^{\prime \prime}-\rho\left(\# \wedge T^{\prime \prime}\right) \circ_{\#} \Gamma_{*}(\langle a, b \triangleleft *\rangle)+\Gamma_{*}(\langle a, b \triangleleft *\rangle) \wedge T^{\prime \prime}$.

As this formula is an instance of formula (30), one deduces from Remark 7.3 that (29) holds in this case.

Third step. Assume now that $T=a$ and that $T^{\prime}$ has root-valence at least 2 . One writes $T^{\prime}=B_{b}^{+}\left(T_{1}, T_{2}, \ldots, T_{k}\right)$. Let $T^{\prime \prime}=B_{*}^{+}\left(T_{2}, \ldots, T_{k}\right)$. In this case, one has

$$
T^{\prime}=T^{\prime \prime} \circ_{*}\left(b \triangleleft T_{1}\right)-\sum_{\alpha} T_{\alpha},
$$

where the sum runs over terms of smaller root-valence.

One then defines $\rho\left(T \wedge T^{\prime}\right)$ using induction on the root-valence of $T^{\prime}$ by the following formula:

$$
\begin{aligned}
\rho\left(a \wedge T^{\prime}\right)=\rho\left(a \wedge T^{\prime \prime}\right) \circ_{*}\left(b \triangleleft T_{1}\right) & -\rho\left(\# \wedge\left(b \triangleleft T_{1}\right)\right) \circ_{\#} \Gamma_{*}\left(\left\langle a, T^{\prime \prime}\right\rangle\right) \\
& +\Gamma_{*}\left(\left\langle a, T^{\prime \prime}\right\rangle\right) \wedge\left(b \triangleleft T_{1}\right)-\sum_{\alpha} \rho\left(a \wedge T_{\alpha}\right) .
\end{aligned}
$$

As this formula is an instance of formula (30), one deduces from Remark 7.3 that (29) holds in this case.

Fourth step. Assume now that neither $T$ nor $T^{\prime}$ is a singleton.

One then defines $\rho\left(T \wedge T^{\prime}\right)$ by the following formula:

$$
\rho\left(T \wedge T^{\prime}\right)=\rho(T \wedge *) \circ_{*} T^{\prime}+\rho\left(\# \wedge T^{\prime}\right) \circ_{\#} T-T \wedge T^{\prime} .
$$

As this formula is an instance of formula (30), equation (29) holds in this case by Remark 7.3 . 
For example, one can compute in this way that

$$
\rho(\langle a, b \triangleleft(c \triangleleft d)\rangle)=a \wedge b \triangleleft(c \triangleleft d)-c \triangleleft d \wedge b \triangleleft a-d \wedge c \triangleleft(b \triangleleft a) .
$$

Theorem 7.4. The morphism $\rho$ satisfies $\langle\rangle,(\rho(x))=(n-1) x$ for every $x$ in CycPreLie $(I)$, where $n=|I|$.

Proof. This property is easy to check for small $n$. By the proof of Theorem 7.2 , it is then enough to check that this property is preserved by formula (30) in the following sense: if it is true for the terms entering the right-hand side, it is true for the left-hand side.

Applying $\langle$,$\rangle on the right-hand side of (30), one finds$

$$
(|r|+|s|-1)\langle r, s\rangle \circ_{i} t-|t|\langle \#, t\rangle \circ_{\#} \Gamma_{i}(\langle r, s\rangle)+\left\langle\Gamma_{i}(\langle r, s\rangle), t\right\rangle .
$$

This can be rewritten as

$$
(|r|+|s|+|t|-2)\langle r, s\rangle \circ_{i} t
$$

which is indeed the correct value for the left-hand side.

7.4. Proof of the isomorphism. By Theorem 7.2, we therefore have a morphism $(\rho, \psi, \mu)$ from the short exact sequence

$$
0 \rightarrow \text { CycPreLie } \stackrel{\iota}{\longrightarrow} \text { Perm } \odot \text { CycPreLie } \stackrel{p}{\longrightarrow} \text { Reflex } \odot \text { CycPreLie } \rightarrow 0
$$

to the short exact sequence

$$
0 \rightarrow \operatorname{Rela}_{v=1} \stackrel{\delta}{\longrightarrow} \operatorname{PreLie}_{v=1} \stackrel{\pi}{\longrightarrow} \text { Indec }_{\geq 2} \rightarrow 0,
$$

where $\mu$ is defined as the quotient morphism from Reflex $\odot$ CycPreLie to Indec $\geq 2$.

Proposition 7.5. The morphism $\rho$ is injective.

Proof. This follows from Theorem 7.4 .

Note that it is necessary here to work over a field of characteristic zero.

Proposition 7.6. The triple $(\rho, \psi, \mu)$ is an isomorphism of short exact sequences.

Proof. We already know that $\psi$ is an isomorphism (87.1) and that $\rho$ is injective.

To conclude, it is enough to note that the dimensions of the rightmost terms are the same. Indeed, the dimension of $\operatorname{PreLie}(n)$ is the number $n^{n-1}$ of rooted trees on $n$ vertices. Because CycPreLie is the primitive of PreLie, the dimension of $\operatorname{CycPreLie}(n)$ is the dimension of PreLie $(n-1)$, namely $(n-1)^{n-2}$. As the dimension of $\operatorname{Reflex}(n)$ is $n-1$, the dimension of $\operatorname{Reflex}(n) \otimes \operatorname{CycPreLie}(n)$ is

$$
(n-1)^{n-1} \text {. }
$$

On the other hand, the dimension of the indecomposables have been computed in [5]: the dimension of $\operatorname{Indec}(n)$ is $(n-1)^{n-1}$ for $n \geq 2$.

Therefore the dimensions of the leftmost terms coincide. This implies that $\rho$ is an isomorphism, hence the statement.

Corollary 7.7. One therefore has an isomorphism $\mu$ :

$$
\text { Indec } \simeq S_{1} \oplus \text { Reflex } \odot \text { CycPreLie } .
$$

Proof. One just needs to check what happens in arity 1 . The component of arity 1 of the $\mathfrak{S}$-module Indec is just $S_{1}$. 
This equivalence has been proved in [5, Th. 5.3] by a computation of characters. We have obtained here an isomorphism that explains why the characters are the same.

\section{Inclusion of CycLie In Perm $\odot$ CycPreLie}

Let us now define a map $\theta$ from CycLie to Perm $\odot$ CycPreLie.

As Lie is a cyclic operad with CycLie as its cyclic structure, there is a surjective morphism of right Lie-modules

$$
S^{2} \text { Lie } \rightarrow \text { CycLie }
$$

We will first define $\theta$ on $S^{2}$ Lie and then check that it is well defined on the quotient CycLie.

From the morphism of operads $\varphi$ from Lie $\rightarrow$ PreLie, one has a map $S^{2} \varphi:$

$$
S^{2} \text { Lie } \rightarrow S^{2} \text { PreLie. }
$$

This map is a morphism of right Lie-modules.

Let $\ell$ be an element of $S^{2}$ Lie. Then one decomposes its image by $S^{2} \varphi$ in $S^{2}$ PreLie according to the roots of the trees:

$$
S^{2} \varphi(\ell)=\sum_{a, b} \mathbf{c}_{a, b} T_{a} T_{b}
$$

where $T_{\#}$ denotes a tree with root \#. Then one can define

$$
\theta(\ell)=\sum_{a, b} \mathbf{c}_{a, b}(a-b) \otimes\left\langle T_{a}, T_{b}\right\rangle
$$

with values in Perm $\odot$ CycPreLie.

Remark 8.1. Let us note that the image of $\theta$ is contained in the image of the section from Reflex $\odot$ CycPreLie to Perm $\odot$ CycPreLie.

Proposition 8.2. The map $\theta$ is well defined on CycLie.

Proof. One has to prove that the right Lie-sub-module generated by relation (2) is contained in the kernel. This just means that this relation should hold for every triple $\left(m_{1}, m_{2}, m_{3}\right)$ of elements of Lie.

Consider the image of $m_{1}\left[m_{2}, m_{3}\right]$. This is

(50) $\sum_{a, b, c}(a-b) \otimes\left\langle\varphi\left(m_{1}\right)_{a}, \varphi\left(m_{2}\right)_{b} \triangleleft \varphi\left(m_{3}\right)_{c}\right\rangle-(a-c) \otimes\left\langle\varphi\left(m_{1}\right)_{a}, \varphi\left(m_{3}\right)_{c} \triangleleft \varphi\left(m_{2}\right)_{b}\right\rangle$.

Now consider the image of $\left[m_{1}, m_{2}\right] m_{3}$. This is

(51) $\sum_{a, b, c}(a-c) \otimes\left\langle\varphi\left(m_{1}\right)_{a} \triangleleft \varphi\left(m_{2}\right)_{b}, \varphi\left(m_{3}\right)_{c}\right\rangle-(b-c) \otimes\left\langle\varphi\left(m_{2}\right)_{b} \triangleleft \varphi(m 1)_{a}, \varphi\left(m_{3}\right)_{c}\right\rangle$.

Then one can rewrite the previous line using the anticyclic structure of CycPreLie, thanks to (6) and (7), to obtain

$$
\begin{aligned}
& \sum_{a, b, c}(a-c) \otimes\left\langle\varphi\left(m_{3}\right)_{c} \triangleleft \varphi\left(m_{2}\right)_{b}, \varphi\left(m_{1}\right)_{a}\right\rangle \\
& -(a-c) \otimes\left\langle\varphi\left(m_{2}\right)_{b} \triangleleft \varphi\left(m_{3}\right)_{c}, \varphi\left(m_{1}\right)_{a}\right\rangle \\
& +(b-c) \otimes\left\langle\varphi\left(m_{2}\right)_{b} \triangleleft \varphi\left(m_{3}\right)_{c}, \varphi\left(m_{1}\right)_{a}\right\rangle .
\end{aligned}
$$

This is exactly (50). 
Proposition 8.3. The map $\theta$ is injective. The composite map $p \theta$ is also injective.

Proof. Let us prove the first statement.

Let us fix a finite set $I$ and let $x$ be an element of CycLie $(I)$ in the kernel of $\theta$. Let us also choose an element $i \in I$. By Proposition 2.1, there is a unique element $y$ of $\operatorname{Lie}(I \backslash\{i\})$ such that

$$
x=(i, y) .
$$

By definition, one has

$$
\theta(x)=\sum_{j \neq i}(i-j) \otimes\left\langle i, \varphi(y)_{j}\right\rangle,
$$

where $\varphi(y)_{j}$ is the projection of $\varphi(y)$ on the span of trees with root $j$. The hypothesis $\theta(x)=0$ implies that for every $j \neq i$,

$$
\varphi(y)_{j}=0 .
$$

So we obtain

$$
\sum_{j \neq i} \varphi(y)_{j}=0=\varphi(y) .
$$

But $\varphi$ is injective; hence $y=0$ and $x=0$.

The second statement follows from Remark 8.1

\section{Inclusion of CycLie In PreLie $v=1$ And CONJectures}

Using the previous inclusion $\theta$ and Proposition 7.6, one gets a map $\lambda$ from CycLie to PreLie $_{v=1}$, hence to PreLie.

Proposition 9.1. The map $\lambda$ is injective.

Proof. This is because $\lambda$ is the composition of $\theta$ (injective) and the isomorphism $\psi$ from Perm $\odot$ CycPreLie to PreLie $_{v=1}$.

Let $M$ be the sub-operad of PreLie generated by the image of CycLie by $\lambda$ in PreLie.

Conjecture 9.2. The sub-operad $M$ of PreLie is a module of generators of PreLie as a free left Lie-module, i.e.

$$
\text { PreLie } \simeq \text { Lie } \circ M
$$

and

$$
M \simeq \text { Indec } .
$$

Conjecture 9.3. The sub-operad $M$ of PreLie is isomorphic to the free operad on CycLie, i.e.

$$
\text { FreeOp }(\text { CycLie }) \simeq M .
$$

In the direction of Conjecture 9.3, Loday and N. Bergeron [2] have proved that the sub-operad of PreLie generated by $x \triangleleft y+y \triangleleft x$ is free. The element $x \triangleleft y+y \triangleleft x$ is the image by $\lambda$ of the element $(x, y) \in$ CycLie. 
Let us summarise these conjectures in words. The image of the inclusion of CycLie in PreLie should generate a free sub-operad of PreLie. This free sup-operad should give a distinguished set of generators of PreLie as a left Lie-module. One should therefore have a canonical isomorphism

$$
\text { PreLie } \simeq \text { Lie } \circ \text { FreeOp }(\text { CycLie })
$$

and an isomorphism

$$
\text { FreeOp }(\text { CycLie }) \simeq \text { Indec } \simeq S_{1} \oplus \text { Reflex } \odot \text { CycPreLie },
$$

where the last isomorphism is Corollary 7.7.

Let us explain now how Conjecture 9.3 would follow from Conjecture 9.2 by an argument of dimension.

Indeed, there exists a surjective morphism of operads from the free operad $F$ on CycLie to $M$, as $M$ is generated by CycLie. To prove that this is an isomorphism, it is enough to compute the generating series. On the one hand, the generating series $f_{M}$ of $M$ is defined by

$$
f_{\text {PreLie }}(x)=f_{\text {Lie }}\left(f_{M}(x)\right)=-\log \left(1-f_{M}(x)\right),
$$

and therefore one has

$$
1-f_{M}(x)=\exp \left(-f_{\text {PreLie }}(x)\right) .
$$

On the other hand, the generating series of the free operad $F$ on CycLie is the unique solution with zero constant term of the fixed-point equation

$$
f_{F}(x)=x+f_{\text {CycLie }}\left(f_{F}(x)\right)=x+\left(1-f_{F}(x)\right) \log \left(1-f_{F}(x)\right)+f_{F}(x)
$$

which amounts to

$$
x=\left(1-f_{F}(x)\right)\left(-\log \left(1-f_{F}(x)\right) .\right.
$$

One can then use the equality

$$
f_{\text {PreLie }}(x)=x \exp \left(f_{\text {PreLie }}(x)\right)
$$

to show that

$$
x=\left(1-f_{M}(x)\right)\left(-\log \left(1-f_{M}(x)\right)\right.
$$

and deduce from this that one must have $f_{M}=f_{F}$.

Remark 9.4. If these conjectures are true, they give a way to define a bigrading on the vector spaces $\operatorname{PreLie}(n)$, where one degree comes from the free operad structure and the other from the free Lie-module structure. The associated generating functions in two variables seem to be related to classical polynomial analogues of $n^{n-1}$ and combinatorial statistics on rooted trees considered for instance in 8 .

\section{ACKNOWLEDGMENT}

The author benefitted from the support of project PEPS "Calcul moulien et MuPAD-Combinat". 


\section{REFERENCES}

1. Nantel Bergeron and Muriel Livernet, A combinatorial basis for the free Lie algebra of the labelled rooted trees, J. Lie Theory 20 (2010), no. 1, 3-15. MR2667815 (2011e:18018)

2. Nantel Bergeron and Jean-Louis Loday, The symmetric operation in a free pre-Lie algebra is magmatic, Proc. Amer. Math. Soc. 139 (2011), no. 5, 1585-1597. MR2763748 (2012b:17001)

3. John C. Butcher, Numerical methods for ordinary differential equations, John Wiley \& Sons Ltd., Chichester, 2003. MR1993957 (2004e:65069)

4. Frédéric Chapoton, On some anticyclic operads, Algebr. Geom. Topol. 5 (2005), 53-69 (electronic). MR 2135545 (2006a:18008)

5. 53 (2010), no. 3, 425-437. MR2682539 (2011g:17014)

6. Frédéric Chapoton and Muriel Livernet, Pre-Lie algebras and the rooted trees operad, Internat. Math. Res. Notices (2001), no. 8, 395-408. MR1827084 (2002e:17003)

7. James Conant and Karen Vogtmann, On a theorem of Kontsevich, Algebr. Geom. Topol. 3 (2003), 1167-1224. MR2026331 (2004m:18006)

8. Dominique Dumont and Armand Ramamonjisoa, Grammaire de Ramanujan et arbres de Cayley, Electron. J. Combin. 3 (1996), no. 2, Research Paper 17, approx. 18 pp. (electronic), The Foata Festschrift. MR.1392502 (97f:05006)

9. Loïc Foissy, Finite-dimensional comodules over the Hopf algebra of rooted trees, J. Algebra 255 (2002), no. 1, 89-120. MR1935036 (2003j:16047)

10. Murray Gerstenhaber, The cohomology structure of an associative ring, Ann. of Math. (2) 78 (1963), 267-288. MR0161898 (28:5102)

11. E. Getzler and M. M. Kapranov, Cyclic operads and cyclic homology, Geometry, topology, \& physics, Conf. Proc. Lecture Notes Geom. Topology, IV, Int. Press, Cambridge, MA, 1995, pp. 167-201. MR 1358617 (96m:19011)

12. Eric Hoffbeck, A Poincaré-Birkhoff-Witt criterion for Koszul operads, Manuscripta Math. 131 (2010), no. 1-2, 87-110. MR2574993 (2011c:18013)

13. Maxim Kontsevich, Formal (non) commutative symplectic geometry, The Gel'fand Mathematical Seminars, 1990-1992, Birkhäuser Boston, Boston, MA, 1993, pp. 173-187. MR.1247289 (94i:58212)

14. Martin Markl, Cyclic operads and homology of graph complexes, Rend. Circ. Mat. Palermo (2) Suppl. (1999), no. 59, 161-170, The 18th Winter School "Geometry and Physics" (Srní, 1998). MR 1692267 (2000g:18009)

15. Martin Markl, Steve Shnider, and Jim Stasheff, Operads in algebra, topology and physics, Mathematical Surveys and Monographs, vol. 96, American Mathematical Society, Providence, RI, 2002. MR1898414(2003f:18011)

16. Christopher R. Stover, The equivalence of certain categories of twisted Lie and Hopf algebras over a commutative ring, J. Pure Appl. Algebra 86 (1993), no. 3, 289-326. MR.1218107 (94e:16031)

17. Bruno Vallette, Homology of generalized partition posets, J. Pure Appl. Algebra 208 (2007), no. 2, 699-725. MR2277706 (2007m:18010)

18. Ernest B. Vinberg, The theory of homogeneous convex cones, Trudy Moskov. Mat. Obšč 12 (1963), 303-358. MR0158414 (28:1637)

Institut Camille Jordan, Université Claude Bernard Lyon 1, 21 Avenue Claude Bernard, 69622 Villeurbanne Cedex, France 